\title{
Copula is an intuitive predicate of consciousness on fulfilment of knowing and judging acts
}

Kiran Pala (iD ${ }^{1 \times}$

The recent investigations into knowledge and its elements viz facts, skills and objects have become prominent in various subfields of philosophy and other areas like linguistics, cognitive science, neuroscience and artificial intelligence. These investigations have been mainly on understanding the relation between the intentionality and its referential entities to know how they enrich knowledge with their existence. This article starts with an exploration of the fundamental aspects of judgemental sense from the knowledge origins perspective. To explain the consequences of this, I start with reconstructing the notion of categorial intuitions described in Husserl's sixth Logical Investigations by relating it to 'knowledge origination' as an intuitive fulfilment of signifying acts. This offers a relation of perceptual synthesis, operating implicitly among the signifying intentions upon grasping intended objects, in view of language structures such as copulas. This leads to the synthesis of intuitive aspects mainly from the categorial elements for a judgemental sense or thinking and the attitude from the very sphere of perception. The tension between categorial intuitions is understood as foundational acts and founded acts. Husserl's sixth Logical Investigation describes the notion of passive synthesis, the genesis of categoriality in every realm of sensibility that allows one to formulate the relational role among the 'act of knowing' and phenomenological approaches to the judgemental sense. Thus, the investigation focuses on perceptual judgements and categories, which fulfils the judgemental sense of categorial elements and attitude.

\footnotetext{
${ }^{1}$ Helsinki, Finland. ${ }^{凶}$ email: kiranap@gmail.com
} 


\section{Sensibilities in judging and act of knowing}

$\mathrm{n}$ history, the systematic relationships of knowing and judging have not spelt out with remarkable exceptions of what we find in Kant's or Husserl's analysis (Guilhermino, 2017; Bellucci, 2019). As a result, there is no consistent basis for a scientific demarcation of hidden goals of thinking, where goals are broader than identifying, locating and recognition, and so on. But, in the cognitive turn, the intuition, imagination, and insights have been illustrated in the systematic framework of thinking processes. However, the recall and judgements are intrinsic combined processes of different modes for 'knowledge grasping or gaining through perceptual acts' (Husserl, 2001a).

It is a phenomenological fusion and signification for explanations on the intentional role of categorial intuition, especially in the analysis of knowledge advancement. According to Husserl, real or genuine knowledge is presented as the result of a synthesis between 'signification and intuition' which are two kinds of intentional acts (Husserl, 2001a). That is, the objective act, at first, intended merely with 'empty' meanings, later becomes perceptible with its intuitive richness: The signifying intentional experiences are fulfilled by intuitive contents. Thus, for example, 'knowing' is characterised as a foundational act which has been built upon a combination of different simple acts (Husserl, 2001a). One should be careful here about where and how the foundational acts will constitute a new intentional object that offers a new objective goal which is not grasped by the simple acts involved.

According to Husserl, the nominal (naming) classification is an example of knowing within which a word or an expression that refers as a name to something serves the job of fulfilment of referring through an intuitive apprehension of the named or referred object ${ }^{\mathrm{l}}$. As a result, at the objective end, knowing is neither signification nor sensible contents, but to a certain extent it is an 'intuited object' of an intuition that reflects in our language expressions. And givenness of such intuition fulfils the signifying intentions: It ensures the evidence of knowledge (Mulligan, 2004).

The same passion is applicable to complex cases of 'knowing' where the judgemental or propositional senses are part of givenness. Such intuitions fulfil the signifying intentions which ensure the evidence of knowledge. For example, in the case of nominal classification, the judgemental sense contains grammatical expressions in which different categorial functions are in action: If a judgement ' $\mathrm{A}$ is $\mathrm{p}$ ' operates under both the categorial functions of the 'subject and predicate', then the statement is a structure of predicative form with the copula, that is, 'is'. Eventually, to express the evidential knowledge as a judgement, it must require a fulfilment of synthesis among the intentional experiences of the foundational acts ${ }^{2}$ that are involved. In this relevance, categorial intuition says 'signifying intentional experiences includes the logical articulations' (Husserl, 2001a); an intuition offers fulfilment to categorially complex intentions.

In particular, the categorial intuition satisfies perceptual judgements of the type 'A is p', the knowing sources. On this notion, we maintain intuitive apprehension as the general scheme of fulfilment for complex judgements, especially in the analysis of nominal classification.

Over the decades several researchers have contributed to the general function of categorial intuition within the sixth investigation-notable among them are Sokolowski (1981), Lohmar (2002), Moran (2005), Benoist (2008), Mohanty (2011), da Silva (2017). In addition, thinkers have usually contributed to 'Formal and Transcendental Logic', 'Experience and Judgment', and 'Analyses concerning Passive and Active Syntheses'. For Husserl, the latter of the two for all such terms are of particular relevance, and hence he has reformulated his analysis on knowing in terms of evident knowledge, particularly in a few important aspects regarding the role of perception in cognitive penetrations with experiences. As a result, this notion describes how our categorial functions of judgements have grounded in our perception (Welton, 1982; Mohanty, 2011; Staiti, 2018; Sato, 2019).

In this context, it is significant to explore the intrinsic aspects of categorial intuition in the presence of perceptual analyses where the sense of judgements is grounded, as this is also a synthesis process of active and passive acts as part of knowledge origination. I have shed light on this to analyse it in relation to Husserl's ideas, given that such an analysis confronts with later descriptions of perceptual analysis of categorial intuition when it loses its epistemic function (Mohanty, 2011). To present it, I follow Husserl's conception of knowledge as expounded in the course of perceptual analysis as part of his sixth Logical Investigation: 'Analyses Concerning Passive and Active Synthesis' (Husserl, 2001a). Although a few critics have confronted it as an alternative conception (Bobrova and Pietarinen, 2019; Staiti, 2015; Theodorou, 2015), I deem that it is a part of the same analysis with few gradual modifications. Importantly, I do not recreate Husserl's position differently; rather, I undertake an analytical key which clarifies the sources (origins) of knowledge proposed in the logical investigations for synthesised comparison of two distinct positions constructed here. In the backdrop, I describe the conception of categorial knowledge containing the sense of judgements that are grounded in perceptual intentions as intentional experiences. It offers a platform to understand the reason underlying categorial intuition.

\section{Components of categorial intuition in Husserlian investigations}

Starting with $\$ 45$ in the sixth Logical Investigation, we look into how the categorial intuition is a foundational act and what are the grounded components in it. To understand, the structural judicative essence in evidential knowledge requires an expansion of the 'sphere of sensibility': (\$45) '[T] here must at least be an act which renders identical services to the categorial elements of meaning that merely sensuous perception renders to the material elements' (Husserl, 2001b, p. 280), that is, a simple act that shapes quality of perception which provides intuitive fulfilment for signifying elements, and those are not completely necessary to articulate logically; for instance, the term 'sky' and the simple intuitiveness expresses the colour of it as 'blue'. Although the expressed judgement 'the sky is blue' and the copula 'is' manifest as the key to evident knowledge for judgement, the categorial forms cannot be fulfilled by these simple intuitions as they are not complete sensible objects which can be perceived immediately. In other words, to be in categorial forms, objects and its constituent parts including relations between them are intended to be articulated together and never be isolated (Sokolowski, 1981; Centrone and Minari, 2017).

As per Husserl, the categorial relations can be intuitively apprehended, and are not considered just to be pure meanings: the object with these categorial forms is not merely referred to, as in the case where meanings function purely symbolically, but it is set before our very eyes in just these forms. In other words: it is not just thought of, but intuited or perceived... what shall we call the correlate of non-sensuous subject-presentation, one involving the non-sensuous structure, if the term 'object' is not available to us? How shall we speak of actual or direct givenness, if the term perception denied us? (Husserl, 2001b, p. 280).

As described in $\$ 45$, the evident knowledge of categorial intuitions and its formed objects expressed by judgements needs intuitive fulfilment for categorial relations. And the sensibility sphere expands in terms of the direct givenness of sensible 
content. This is a kind of givenness that does not exhaust the realm of sensibility, because of the intuitional fulfilment for the categorial forms of signifying intentions in judgements. The desire is to obtain a categorial intuition, and Husserl believes that evident judicative knowledge would be unachievable without a specific intentional act (Mohanty, 2011). In contrast, $\$ 46$ and $\$ 47$ investigations say that categorial intuition placed at a higher level and ordinary sense-perception deals with simple sensible intuitions directed to real sensuous objects ${ }^{3}$.

It is also significant to note that sensible intuitions are simple as they do not use any conditions of distant previous acts to present an objective pole at an end, because of the absence of intentional foundations. And, it operates on a single act-level which does not contain, or has not been constructed on, any previous layers of acts. In contrast, categorial intuition turns on the opposite because of its sensible intuitions being the foundational acts that contain both previous and later objective poles that have both formed and sensible objects. However, for Husserl, formed objects are new, and that appears only through respective categorial acts ${ }^{4}$. Thus, I pursue the line of thinking that categorial intuitions can be seen as epistemically dependent on simple intuitive acts, and no formed objects have yet been apprehended there. However, without sensible apprehension of objects the formation of categorial intuitive apprehensions of former objects would not have been possible. Therefore, this complicates the basis to have Husserl's analysis of knowledge in terms of categorial intuitions.

Husserl emphasises that knowing is the combination of intuition and signification which are two root intentional acts understood as foundational acts. However, at least with judicative knowledge, also considered to be propositional knowledge, there is an additional internal level which is the very intuition, a sort of condition fulfilling the signifying intention. This makes categorial intuition represent itself as intuition in part of the 'act of knowing'. As in the formation of a categorially articulated intuition, knowing is an act which is distributed between two levels of epistemic foundations: the primary level of knowing, constructed through a fulfilment of the combination of a signifying intention and an intuitive intention, and the secondary level of construction that moves from the internal to the global, that is, when categorially articulated the intuition fulfilment is founded on both simple sensible and categorial intuitions (which are themselves founded on simple intuitions). Now I shall focus on the foundation which makes the categorial intuitive act possible, with reference to $\$ 47$, which also provides a characterisation of the simple sensible intuition in view of a correlation between partial perceptual intentions that present their intended objects and the fusion of sense-contents. As a result, the simple intuition acquires sensible objects in isolated conditions, but the intuition is not an isolated act, because it has not been discontinued from any of the perceptual intentions through partial grasping of intended objects.

As in $\$ 46$, multiple partial intentions compile the perception of a single isolated sensible object and the fundamental functionality of perception remains unchanged, as there are no new acts that stand differently in the intentional foundations. It means, the partial acts of perception have not synthesised in the sense of a new intentional act, but they are fused into a homogeneous object $^{5}$ at the same intentional level. And, the other character of simple intuition is particularity, since the sensible object is intended in its unity, though it requires different partial intentions in constant fusion for grasping onto certain aspects of the object. Thus, 'in sense-perception the 'external thing' appears in one blow, as soon as our glance falls upon it' (Husserl, 2001b, p. 283). It implies that partial intentions are always fused into a global intuitive object, as the grasped sensible object continuously perceives the same as a single object (Husserl, 2001b, p. 284).
As literature states, in a simple sensible intuition, our perceptual intentions are always being directed to the object in unity. For instance, 'whether I look at this book from above or below, from inside or outside, I always see this book. It is always one and the same thing, and that not merely in some purely physical sense, but in the view of our perceptions themselves' (Husserl, 2001 b, p. 284). Thus, the 'whole' is an object which is immediately perceived, although, the sense-perception involves a fusion of several partial apprehensions. So, the global perception of the object constantly imposes itself on the perceived reality which reflects a simple perception. It is through the relation between global perception and the partial perceptual intentions, when fused, ${ }^{6}$ that the categorial intuitions will manifest. The state of this fused synthesis with intuitive fulfilment provides categorial articulations. Hence, it is possible to observe this in the appearance of partial sense-intentions and their combination with global perception, especially in Lohmar's interpretations (Lohmar, 2002).

On the same plane, $\$ 48$ states that upon a perceived object, the intuitive character of the judicative intention ' $\mathrm{A}$ is $\mathrm{p}$ ' has formed, and it turns out that an act of perception comprehends the object ' $A$ ' as a whole in universals at a blow. As a dependent moment, an act of perception has trained upon ' $\mathrm{p}$ ' which belongs to object ' $A$ ' relatively. These two acts are not performed together, instead one after another, in a way of disjoined experiences. Otherwise, they together would not bind as if in a single act where the object ' $A$ ' contains ' $p$ ' within. Thus, the disclosure of partial intentions which intend the aspect ' $p$ ' belonging to the object ' $A$ ' is implicit and synthesised with global intentions, and it is dependent on the moment object 'A'. Such a synthesisation process is sometimes also known as a deeper way of being a predicative categorial relation, given explicitly but intuitively fulfilled. This phenomenon is regardless of whether categorial intuitions are presented on simple intuitions to which they cannot be reduced ${ }^{7}$ to any other. Categorial intuitions appear in a new sort of independent act that has its own perceptual objects. In fact, Husserl touches on this point in $\$ 48$ in terms of fusing our total perception with one specific perception: '[In] the narrowing down of our total perception to one specific perception, the part-intention to " $p$ " will not be torn out of the total appearance of A, so as to break up the latter's unity, but an independent act will have " $p$ " as its own perceptual object. At the same time, one's continuously operative total perception will "cover" this specific perception in respect with one implicit part-intention.' (Husserl, 2001b, p. 287).

What is interesting here is that an emphasised aspect of object ' $A$ ' is a particular perceptual intention which correlates with the constitutive aspect ' $p$ ' as it appears explicitly in global perception; the event of 'covering' in synthesis takes place among intentions. Now onwards, the object of global apprehension will determine through the emphasised partial aspect. If there is a covering synthesis between partial and global apprehensions, then the unity of perception provides the categorial intuitive content as a continuation to the covering synthesis process. Thus, categorial intuition should be considered to be the unification of explicit partial intentions with a global perception of the intended objects, rather than merely sensible intuitions. As a result, a 'covering unity' depends on what provides intuitive fulfilment for categorial forms. That is, the categorial aspects of the founded intuitions have a kind of sensible content collected through simple intuitions as caused by the covering synthesis between partial intentions and global perception. However, this kind of synthesis guarantees the categorially articulated signifying intentions: how categorial intuition occurs from 'covering unity', 8 and an unfamiliar object appears within the state of affairs. This is similar to those relations we expressed in judgements, such as a predicative relation 'A is $\mathrm{p}$ ' with the copula 'is', and they can only give 
intuitively through the covering synthesis between partial intentions and simple global intentions, which leads to another intuition.

Here one should observe that the two-folded foundation in intuition constitutes the evident judicative knowledge that is covered with structural differences. The foundation of categorial intuition needs a covering synthesis, making it evident that it is a synthetic type and is different from simple intuition. The synthesis with the more autonomously relative character has the capacity of continuous fusion of contents. In categorial intuition, partial intentions are implicit. Later they become explicit and get overlaid onto the global perception, where partial intentions are intrinsic. In a strict sense, a covering synthesis of intentional experiences takes place on the act of knowing on which the synthesis obtains between categorial intuitions and significations (empty intentions). Importantly, it does not seem accurate, though this is to understand the nature of fulfilling synthesis at the base level of categorial intuition.

Whatever way this turns out, the partial intuitive content correlates with the global givenness of the object which ensures that the sense of an object in parallel fulfils the intuitive content at a higher plane. The concept of fulfilment involves the formulation of empty intentions and the intuitive content covered with experiences. It is the principle characteristic of the act of knowing (synthesis) between empty intentions (significations) that offers fulfilment to the intuitions: a covering synthesis between significations and intuitions. The notion of acts brings out coveringness of the experiences in the intuitive givenness. Until then, they intend symbolically. As a result, covering has been considered to be the fulfilment for synthesis between significations and intuition. However, it is worth discussing the two-folded or double synthetic structural relation as foundation layers within the act of knowing. It appears that the characterisation of knowing takes place only at higher-level relations, specifically at intentional modalities, not in categorial intuitions. We shall discuss this further in the following section, but before we proceed, it is important to understand the aspects of intuitions and thoughts in view of the 'act of knowing' with reference to Logical Investigations.

\section{The realm of intuitive acts and meaning in Husserlian investigations}

As Husserl describes, significations are the empty intentions themselves, and how they fulfil intuitively with the notions of categorial intuitions needs an advancement. Knowing that, intuitions derive autonomously from the thought acts and they just operate through categorially formed significations. It is evident that the realm of meaning does not restrict itself to the origins of categorial intuitions as mentioned in $\$ 63$ of Logical Investigations: ' $[\mathrm{T}]$ he realm of meaning is [...] much wider than that of intuition, i.e., than the total realm of possible fulfillment' (Husserl, 2001b, p. 312). It declares that pure thought is independent of intuition. Significations are open to combining themselves freely, regardless of what the intuitive restrictions are. As a result, the signifying acts refer to purely logic-grammar laws (for instance, language structures) rather than mere intuitions, which sets the possibilities for sense-bearing statements as argued by Renaudie (2013). The intuitive content does not by itself keep any categorial relations, but this content can at most be a function of confirmation for categorial articulation which originated from the pure thought sphere. For instance, Husserl states in $\$ 62$, '[W] ith real [intuited] contents none of the categorial forms which fit them is necessarily given: there is abundant freedom to connect and relate, to generalise and subsume, etc.' (Husserl, 2001b, p. 309). The key argument is that intuition contributes to only the situations for the evident confirmation of logical articulations not originally stemming from the intuitive, and it does not serve to the creation or ordering of categoriality by itself. So, there is a discontinuity between intuitions and thoughts, which is a serious concern for the genesis of creation and operation of categoriality. This is mainly because categoriality requires in advance a set of logical functions through pure thought orders which can express itself without external restrictions (Sokolowski, 1981; Manca, 2019).

It seems necessary to deem that intuition is a constitutive part of knowing and judging, not just a bearer of categoriality. It has the power to affirm and dis-confirm the categorial relations produced by pure thinking. This conclusion is applicable merely to sensible objects in isolated conditions carried over by simple perception, not by any relations between the constituent parts. According to Husserl, categorial intuition is depicted as something drawn from the evident apprehension of isolated objects and logical relations. We can observe this argument in simple sensible intuitions, as there are multiple partial intentions fused together and do not appear explicitly as they are: the general appearance of the intended object as a 'whole' in global perception through simple sensible intuitions. As we have observed, categorial intuitions derived from the disclosures of certain partial intentions and their covering synthesis is with global perception. So, the intuitiveness applies to all kinds of relations when they are ready to forge as a judgement and it gives distinct types of relations.

The intuitiveness of categorial intuitions can establish itself as an intuition, such that it will offer evidence to judicative categorial relations. However, the source of evidence offers nothing other than the characterisation of an intuition by something intentionally different from it. In any case, it is all about the perspectival intuitive content, moving from which the categoriality of signification delimits the ways of how it is synthesised into categorial intuition. For instance, according to Benoist (2008), ' $[\mathrm{I}]$ $\mathrm{n}$ knowing, intuition is molded according to signification or, in other words, intuition operates in exclusive accordance with that which signifying intentions set up as a target to be attained' (Benoist, 2008, p. 211).

To consider, a categorial intuition is a foundational act where the process is guided by signifying intentions. To fulfil this condition, it requires an explicit synthesis of implicit partial intentions with global perception. This direction turns to where certain categorial relations gain confirmation from perceptual intentions, which are partial, for example, a sentence: 'The apple is red'. In this case, the categorial functions have been guided for the act of knowing: partial intentions which are perceptual, highlighting 'the colour of the object' (which is red), instead of emphasising other factors such as shape, texture, taste or quality, etc.-these are explicit and synthesised with the global apprehension of the object, 'a red apple'. It is because simple intuitions are attentive to relations in virtue of signifying intentions that carry intuitiveness, where simple intuitions have been synthesised in a specific manner to provide intuitive fulfilment for the categorial articulations at work.

Logical articulations derive from pure categorial thinking expressed by judgements, but not from the intuitive content which is used for the fulfilment. And these logical articulations are fully formed before the confirmation of their intuitiveness, and their groundedness guides the selection of the partial perceptual intentions, for the synthesisation in categorial intuitions. This way, intuitions have been molded by the categorial relations which are expressed in the judgements; for instance, as a stake from the stakeholder statements. In fact, the intuition allows itself the categoriality that characterises categorial intuitions, when the relevant logical relations are explicit and independent of 
judicative intentions. According to Husserl, 'categorial articulations are originally exterior to intuitions', and later they become functional knowing, but to verify this at first offers no clear connection to the evidence of logical relations.

Nevertheless, this sixth Logical Investigation says that intuition accepts logical restrictions from judgements to enrich meaningful certainty. If we pursue this line of thinking, a simple partial perceptual intention will be created explicitly instead of other factors. It is the revelation of the importance of signification (empty intentions) in the knowledge ordering as seen in the example of 'the red apple' structure. But, how intuition guides through logical relations originally and on what grounds this prevails are not still clear. I presume that there must be some continuity among the categorial understanding and sensibility, and it might be a prior act for the intuition concerned regarding the categorial forms. Also, if there is no continuity among these two realms concerning origins and functions of categorial relations, it would be unclear as to why intuitive acts are led by complex structures outside of their original functioning. This will be discussed in the following sections with the case of perceptual analysis from Husserl's point of view.

\section{Perceptual synthesis for judgements}

It is important to understand Husserl's conceptual change about categorial intuitions as mentioned in 'Perceptual Analysis for Passive and Active Synthesis' (APAS). I pursue APAS as an extensive reflection on logic as it is the theory to justify knowledge (please see Husserl, 2001a, pp. \$1-10). I consider it as a further extension of Logical Investigations itself.

Husserl says that the genetic method is an approach to explain how the subjective sources guarantee the evidential givenness with implicit logical validity. In kind, it is vital to see how subjectivity arises from founding (primary) constituent layers to make explicit the requirements of theoretical thinking. Also, it must correlate to its objective scope where its foundations are in pre-theoretical synthesis that are turned to the sensible world as an intuitive function. The conceptual activity determines pretheoretical life of consciousness and its intuitive function, where the thought brings out scientific understanding of knowledge (Mohanty, 2011). We may thus look into how a process takes place to root in sensible experience as the epistemic ground for having higher-level intentional acts and its relations.

This section explores merely a few insights to understand the role of categorial intuition in APAS, rather than venture to spell out exhaustive analysis. So, Husserl's reconsideration of the differences between the two levels of knowledge foundations are key concerns. The general structure of knowing and their salient characteristics are presented through the intuitional sphere. For instance, Husserl described 'perceiving as a two-stage process': '[T] he object is constantly given to us as unchanged, as the same (Husserl, 2001a, p. 34), And then there is a constant variation of modes of appearance, perspectives, so that we have a consciousness that runs through them and connects them up, a consciousness of the one and the same object' (Husserl, 2001a, p. 34). This has been mentioned in $\$ 10$, the introduction of APAS. Here, 'perceptual awareness' emphasised as a characteristic of perception is the constitutive relation between the unitary givenness of the apprehended object and its diverse modes of appearances that remains unchanged.

Husserl says that the modes of appearances are explicit and their aspects are complementary to experiences. As a result, when the perceptual process unfolds, the initial part establishes itself as an identity of the perceived content: recognising objects and later being intended. In fact, we view them as a single object from a series of variable perspectives. For instance, seeing an object with subjectivity with an objective scope, the difference is all about how the content establishes in the world's sense and how that appears in the judicative evidential knowledge. Let's now come back to the case of perception. An identifying synthesis of the partial modes of appearance or manifestation of an object allows for the object recognition process: determining the identity of the perceived object. I posit that its general schema is similar to the content fusion in simple perception, as mentioned in the sixth Logical Investigation.

Husserl renders that the temporal flow of partial intentions as the structural gain and loss of intuitive prominence is an apprehension process. It becomes clear that the partial modes of givenness inherent in an object's identity are not just partially apprehended, but they are also successive modes in terms of an ordered structure in a temporal flow. When these modes give up their identity to other modes of givenness, till then they are only suggestive modes of givenness for a new apprehension; thus, they have been perceived attentively (Steinbock, 1998; Husserl, 2001a). The identities of perceived objects are constituted and manifested in distinct aspects which are part of consciousness and become structurally inherited. This is how a single object is perceived with multiple modes of givenness in terms of manifestation or appearance. It is an activity secured when all these modes are not available to consciousness, and this is a phenomenon that explains why only the structural succession is available for consciousness. To be precise, let's have a look into Husserl's description of the perceptual process 'between what is genuinely perceived and what is not genuinely perceived' (Husserl, 2001a, p. 40). We can observe that every single perceptual intention has a particular aspect (second) of an object and is emphasised with certain intuitive features, mainly temporal apprehensions (present, past, and future). Later, they become the central theme of perceptual consciousness, because the intuitive features are embodied in perceptual grasping of an object. This shows that perceptual synthesis is not just a fusion of intuitive contents added one to another effectively. It is also a confluence of both aspects: what has been given intuitively and not given (cointended) in every object perspective. The synthesis is between distinct types of perceptual intentions, some of which are effectively intuitive, and some others are not very intuitive which confers the objectual global identity of the perceived object. For example, in connection with universals extracted from particulars through intuition as in 'the sky is blue' argument, the genuine intuitive knowledge of colour of the 'sky' is taken to be 'blue'. However, the non-intuitive knowledge constituting the potential colours of the 'sky' may be orange, violet etc. across VIBGYOR. Therefore, perceptual synthesis is not merely the convergence of the intuitively given and non-given. It is also the consolidation of foundational layers of the varied perspectives of the object 'sky' which constitutes an objective global identity. These remarks throw light on the relations of recent knowledge in its origins and nature.

So, the intentions are fully intuitive, and the synthesis with others is merely additional aspects of intuitiveness. Those 'intuitive intentions' are part of the intuitive givenness with the aspects referred to (and by) the intuitive data at a certain given moment, as already perceived or as newly perceived. As a result, the object in perceptual apprehension always goes beyond the particular intuitive grasping moment, since the relevant aspect of apprehension is not yet apprehended in their intuitive fullness, but merely influenced by what has been perceived already or prospective apprehension. For instance, '[I]t is clear that a nonintuitive pointing beyond or indicating is what characterises the side actually seen as a mere side, and what provides for the fact that the side is not taken for the thing, but rather, that something transcending the side is intended in consciousness as perceived 
(Husserl, 2001a, p. 41). In each turn, the partial data have intuited effectively, but never considered them as whole objects in being identical with it, rather merely synthesised with the horizons of further doable data. So, the objects perceived with their global character can achieve apprehension through the constitutive partiality of each perceptual givenness. For instance, it is evidential knowledge that there are varied appearances of the object 'sky', but the form still arises from a non-intuitive perspective which exists as the earth travelling across the orbit changes its colour from blue to a reddish hue depending on the distance between the sun and the earth.

In Husserl's view on the passive ordering of perception, for higher-order synthesis posited between two intentional acts: signification and intuitions constitute a prominent affair. It revolves around identifying certain structures earlier attributed only to the relations existing between intentional modalities which are simple intuitions or perceptions ${ }^{9}$. As Husserl says in APAS: '[W]e now have to describe the process of perception as a process of acquiring knowledge' (Husserl, 2001a, p. 44). So, for Husserl, the prominence of a particular feature that defines knowledge is the fulfilment of synthesis. For instance, when he talks about the 'act of knowing', merely perception does not constitute knowledge by itself, and it must require an act of fulfilling synthesis between signification (empty intentions) and intuition (intuitive givenness) which are intentional modalities. These are already in the ordering of simple perception autonomously. In fact, there are empty intentional prefigurations of experiences, inclusively which can fulfil with the aspects of an anticipated act as they comprehend perceptual consciousness. It is very similar to attaining full intuitive givenness. The empty intentions posit gradual fulfilment by formulating the horizon for every single intuitive act. Thus, I contend, the transformation always takes place from the undetermined to fully determined. So, the conceptual activity transforms as knowledge within the premises of simple perception, and without interference of signifying acts. However, the transformation has variance.

\section{Judgements and categories in perceptual realm}

In the earlier sections, the general structure of synthesis in relation to Logical Investigations have a prominent space in simple perception. It shows how simple perception holds the basic categorial articulations; in fact, these articulations are found at an intentional level.

Husserl discusses how 'active synthesis' can constitute the notion of an object, and in the process of knowledge acquisition about the objects or the world perception cannot reduce to a passive event. Substantially, our perception with cognitive interests should consider an initial level of subjective activity. Therefore, it can reveal explicitly the involvement of intrinsic elements in the content: significations (empty intentions) and categorial articulations (Husserl, 2001a). In this active process, Husserl identities are two momentums, which belong to the perceptual realm, understood as the initial acts of intentionality. As far as the judgemental act goes, objectivation is involved effectively with cognitive interests, but it is not that easy to notice this realm through its initial active exercises: genetic and sensible syntheses (Husserl, 2001a). As a result, the judgement expresses that certain knowledge is still considered to be a founded act. But, this act must need intuitions as its founding layer. In other words, the role of intuitions in judgements cannot be neglected, and it is not sensible to regard categorial articulations as external to intuitions in perception.

Originally, the perception should be the establishment of the perceptual theme in the constitution of an objective sense. We can notice this in directed attention which affects the subject matter; for example, when we direct our attention to an unfamiliar sensible object such as an unknown sound or a visual object. It distracts the attention and diverts the subject towards something which attracts us. This change of the attentive state through the cognitive interests in perception is evident. In fact, this is just about what occurs in the object's fixation as the substrate and centre of a unitary interest (Husserl, 2001a, p. 290). It is prior to actual perceptual exploration through cognitive interests. At this primary level, the notion of objects has not fully formed as the objectivity of the apprehended data has just begun. The perceptual theme will gradually be enriched to a degree where it can form as an intentional object and grasped in undifferentiated generality (Husserl, 2001a, p. 293). It has marked the notion of global perception as described in the sixth Logical Investigations when affecting pre-data turns to conceptual data without exhibiting the parts of the object, but as a whole a perceptual theme is being constituted uniquely.

There is always a difference between global perception and simple perception as per the sixth Logical Investigation, because the global grasping functions are implicit until the partial simple intentions become explicit. This happens through a rendering process where the objects are at least some instances. As per APAS, however, it appears only when required by the categorial signifying intentions. This is an undifferentiated apprehension or perception in a perceptual process only for the initial moment, but it differs from the usual character of the perceptual process since it is an autonomous intentional modality.

\section{Conclusion}

We have observed that the general structure of knowing synthesis and perceptual activity follow a genetic ordering. It shows the fulfilment of categorial forms (includes intuitions, articulations, and intentions) which are grounded in synthesis, where perception autonomously explores its themes. So, the categorial intuitions have to be replaced by the perceptual explication process regarding intuitive synthesis. Therefore, the general structure has a genetic continuity, and it flows through an intuitive synthesis between sensibility and knowledge for judgements. And, the significance can direct the intuition to higher-level acts of knowledge for its basic structure which takes place in the notions of unrevealed sensibility.

As discussed, the fundamental problem dealt with opens up the scope to delve into aspects of categoriality and its forms. For instance, global perception shows an intentional modality as just an explanatory process which has been triggered by the initial levels of affection. If one continues with cognitive interests from a genetic perspective, the genetic history of cognitive interests becomes objectivised. Then the global perception loses its autonomy in intentional modality as an objective sense constitution process. There are also further questions like how the covering synthesis and categorial intuitions have grounded themselves in each other in the active mode of perceptual activity, and what categorial forms are inherent in intuitive synthesis infused with cognitive interests. This is still open for further research. Investigations into these questions might provide an advancement in understanding the fundamental aspects of the sensibility of judgement in an 'act of knowing' and acquisition as a conceptual activity rather than as mere causal reasoning. I believe that these arguments from Husserl's sixth Logical Investigations and APAS would lead to further developments of ideas on judicative essence in evidential knowledge at a higher level. Further extensions on this study will be towards the second level of perceptual activity. 


\section{Data availability}

Data sharing not applicable to this article as no datasets were generated or analysed during this study.

Received: 23 May 2020; Accepted: 22 September 2020;

Published online: 12 October 2020

\section{Notes}

1 "The relation, as one of naming, is mediated, not merely by acts of meaning, but by acts of recognition, which are here also acts of classification. The perceived object is recognized for an inkpot, known as one, and insofar as the act of meaning is most intimately one with an act of classification, and this latter, as recognition of the perceived object, is again intimately one with the act of perception, the expression seems to be applied to the thing and to clothe it like a garment" (Husserl, 2001b, p. 202).

2 "[.......... in the case of a perceptual statement, not only the inwrought nominal presentations are fulfilled: the whole meaning of the statement finds fulfillment through the underlying percept" (Husserl, 2001b, p. 271).

3 The characteristics of the objects are: "sensuous objects are present in perception at a single act level: they do not need to be constituted in many-rayed fashion in acts of higher levels, whose objects are set up for them by way of other objects, already constituted in other acts" (Husserl, 2001b, p. 282).

4 It is like the new objects formation based on the older objects along with their relations to what appears in the basic acts (Husserl, 2001b).

5 "The act of perception also is always a homogeneous unity, which gives the object 'presence' in a simple, immediate way" (Husserl, 2001b, p. 284)

6 "The individual precepts of our series have a continuous unity: In this unity, our manifold acts are not merely fused into a phenomenological whole, but into one act, more precisely, into one perception" (Husserl, 2001b, p. 284).

7 When the global perception of the intended object and the partial intentions are fused and operate on the same intentions implicitly under a cover among them.

8 The covering unity between global and partial intentions "will not set itself up as our object, but will help to set up another object. It will act representatively, and to such effect that, A will now appear to contain 'p' in itself (or, with a reversed direction, 'p' will appear as contained in A)" (Husserl, 2001b, p. 287).

9 Sometimes in historical texts, it also refers to simple perceptions considering a matter of difference in terminology (Centrone, 2010).

\section{References}

Bellucci F (2019) Philosophy of notation in the 19th century. In Peirce, Husserl, and All the Others on Inclusion and Assertion, Peirce and Husserl: Mutual Insights on Logic, Mathematics and Cognition. Springer, pp. $61-75$

Benoist J (2008) Linguistic phenomenology? In: Mattens F (ed) Meaning and language: phenomenological perspectives. phaenomenologica. Springer, Dordrecht, pp. 215-235. 187

Bobrova A, Pietarinen A-V (2019) Thoughts, things and logical guidance, Peirce and Husserl: mutual insights on logic, mathematics and cognition. Springer, pp. $43-58$

Centrone S, Minari P (2017) Husserl and Schröder, Essays on Husserl's logic and philosophy of mathematics. Springer, pp. 125-145

Centrone ST (2010) Logic and philosophy of mathematics in the early Husserl. Springer, Dordrecht

da Silva JJ (2017) On color: the Husserlian material a priori, how colours matter to philosophy. Springer, Switzerland, pp. 97-105

Guilhermino DP (2017) Husserl and Brentano on presentations and judgments. Guairacá-Revista de Filosofia 33(2):91-101

Husserl E (2001a) Analyses concerning passive and active synthesis. Kluwer Academic Publishers, Dordrecht and London, pp. 31-36. (trans: by A. Steinbock) 28(29)

Husserl E (2001b) Logical Investigations. Routledge, London/New York, Vol. 1 (Husserliana XVIII) (trans: Findlay, JN)
Lohmar D (2002) Husserl's concept of categorical intuition, hundred years of phenomenology. In Zahavi D, Stjernfelt, F. Springer, Dordrecht, pp. 125-145

Manca D (2019) Syllogism and Mathesis. In Mathesis, Grund, Vernunft (eds) Hegel and Husserl on the Method of Philosophy. Ergon-Verlag, pp. 131-146

Mohanty J (2011) Edmund Husserl's Freiburg years: 1916-1938. Yale University Press, New Haven, London

Moran D (2005) Edmund Husserl: founder of phenomenology. Wiley, New York Mulligan K (2004) Essence and modality. The Quintessence of Husserl's Theory In: Siebel M, Textor M (eds) Semantik und Ontologie. Beiträge zur philosophischen Forschung. Ontos Verlag, Frankfurt, pp. 387-418

Renaudie P-J (2013) Me, myself and I: Sartre and Husserl on elusiveness of the self. Continent Philos Rev 46(1):99-113. Springer, Dordrecht

Sato S (2019) Husserl on experience, expression, and reason. In New Phenomenological Studies in Japan, Springer, pp. 83-94

Sokolowski R (1981) Husserl's concept of categorial intuition. Phenomenol Human Sci 12:127-141. Supplement to Philosophical Topics

Steinbock A (1998) Husserl's static and genetic phenomenology: translator's introduction to two essays. Continent Philos Rev 31(2):127-134. Dordrecht and London, Kluwer Academic Publishers

Staiti A (2018) Pre-predicative experience and lifeworld. Two distinct projects in husserl's late phenomenology. In Oxford handbook of the history of phenomenology. Oxford University Press, Oxford, pp. 155-172

Staiti A (2015) Husserl and Rickert on the nature of judgment. Philos Compass 10 (12):815-827. Wiley Online Library

Theodorou P (2015) Husserl's Doctrine of "Categorial Intuition" and Heidegger's Seinsfrage. Husserl and Heidegger on Reduction, Primordiality, and the Categorial. Springer, Switzerland, pp. 245-294

Welton D (1982) Husserl's genetic phenomenology of perception. Res Phenomenol 12:59-83. Netherlands: Brill

\section{Acknowledgements}

Earlier versions of this paper were presented as K. Pala (2017) at the Mind and Brain Annual Conference, Flint, USA. I would like to thank the audience of the conference for their helpful comments. I also wish to express my gratitude to Cyril McDonnell, Swetha Pala and Vamshi Velagapuri for helpful comments on earlier versions of this paper. I could not have completed this project without encouragement and support of my dearest family members Mrs. Swetha Pala and adorable Agasthi Shri in every moment of my life

\section{Competing interests}

The author declares no competing interests.

\section{Additional information}

Correspondence and requests for materials should be addressed to K.P.

Reprints and permission information is available at http://www.nature.com/reprints

Publisher's note Springer Nature remains neutral with regard to jurisdictional claims in published maps and institutional affiliations.

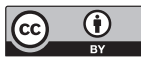

Open Access This article is licensed under a Creative Commons Attribution 4.0 International License, which permits use, sharing, adaptation, distribution and reproduction in any medium or format, as long as you give appropriate credit to the original author(s) and the source, provide a link to the Creative Commons license, and indicate if changes were made. The images or other third party material in this article are included in the article's Creative Commons license, unles indicated otherwise in a credit line to the material. If material is not included in the article's Creative Commons license and your intended use is not permitted by statutory regulation or exceeds the permitted use, you will need to obtain permission directly from the copyright holder. To view a copy of this license, visit http://creativecommons.org/ licenses/by/4.0/

(c) The Author(s) 2020 